\title{
LOS INSECTOS DE LA REGIÓN DE MAGALLANES EN LA RECOLECCIÓN DE LA EXPEDICIÓN DEL PACÍFICO (1862-1865) ${ }^{1}$
}

\author{
THE INSECTS OF THE MAGELLAN REGION FROM THE RECOLLECTION OF THE \\ EXPEDITION OF THE PACIFIC (1862-1865) ${ }^{1}$
}

Vicente Pérez ${ }^{2}$

El 10 de agosto de 1862 partieron del puerto de Cádiz, España, dos buques de guerra de la Armada Española, las fragatas "Resolución" y "Triunfo", a las que se sumaría en aguas del río de La Plata la goleta "Covadonga" para conformar la llamada Comisión Científica del Pacífico (Santos Mazorra, 1994). Al mando de esta división naval iba don Luis Hernández Pinzón y Álvarez, comandante general de la Escuadra del Pacífico (1862-1865) (Martínez Valverde, 1958).

Esta expedición empezó a planificarse en 1860 entre los gobernantes españoles como una operación militar y política que respondía así al clamor de los diplomáticos hispanos en varias repúblicas americanas que consideraban que el envío de buques de guerra era necesario para defender los intereses peninsulares, fortalecer el comercio y estrechar los lazos políticos con las ex colonias españolas.

Añadir una Comisión Científica a la expedición político-militar, tuvo un interés secundario, ya que la recolección de ejemplares de flora, fauna y gea de los países visitados para su estudio y exhibición "tal como la practican los países cultos (Cabodevilla, 1998) era solo un intento del Gobierno para dar una señal de la preocupación científica de España en este aspecto para salir del atraso en que se encontraba, ya que no poseía instituciones tan potentes como el Jardin de Plantes de Paris (fundado en 1626, parte integrante del Muséum National d'Histoire Naturelle de Francia, con sus 22,5 hectáreas, que está bajo el patronazgo de Georges-Louis Leclerc, conde de Buffon (1707-1788) y de Jean-Baptiste- Antoine Pierre de Monet de Lamarck (1744-1829)); o el British Museum of Natural History (fundado en 1753) (Cabodevilla, 1998). Cabe destacar que las instituciones mencionadas no solo se fundaron, sino que se desarrollaron continuadamente en el tiempo, porque en España, si bien en tiempos de Fernando VI (1713-1759) había surgido la idea de la creación de un Museo de Historia Natural, este proyecto solo llegó a concretarse cuando asumió el trono su hermano Carlos III (1716-1788) (Real Decreto del 17 de octubre de 1771). La naciente institución comenzó a acumular diversas especies para su exhibición, pero nuevamente, la falta de interés de las autoridades produjo la decadencia del Museo, entre cuyos

\footnotetext{
${ }^{1}$ Correspondiente al Proyecto Antecedentes para la Historia de las Ciencias Naturales en la Región de Magallanes.

${ }^{2}$ Laboratorio de Entomología, Instituto de la Patagonia, Universidad de Magallanes. Casilla 113-D. Punta Arenas. Chile.
} 
materiales se encontraba "la colección de insectos recogida por la Comisión del Pacífico, colocada en cajas, por localidades y sin estudiar" (Gomis Blanco, 1921).

Se trató de una comisión de una España empobrecida por acontecimientos originados por diversas circunstancias políticas como guerras externas e internas, desmembramiento del imperio de ultramar con su desaparecimiento total al finalizar el siglo y desastres económicos, empobreció a España, la alejó de Europa y mantuvo a los intelectuales, a los universitarios $y$, sobre todo, a los hombres de ciencia, aislados (Alvarado Ballester, 1987). Llegaron a la costa oriental de América, estuvieron en el río de La Plata, visitaron varios puertos de Brasil, pero el problema de estar siempre sujetos a los movimientos de la armada y condenados a su inmovilidad y a sus prisas, hizo que el jefe del grupo de los naturalistas, Patricio María Paz y Membiela, tomara la decisión arbitraria de dividirla en dos subcomisiones: una que proseguiría por tierra, independiente de las naves, y otra que continuaría por mar, adaptándose a las órdenes del Ministerio de Marina. Éstas prescribían que solo la fragata Covadonga podía ingresar al estrecho de Magallanes para realizar algunas observaciones hidrográficas.

Tensiones generadas entre algunos miembros de la expedición y jefes y oficiales de la Armada, hicieron que el jefe de la expedición, Patricio María Paz y Membiela, dispusiera arbitrariamente la división de ella en dos grupos: uno que proseguiría por tierra y otro que viajaría por mar. Esta última subcomisión zarpó de Montevideo el 16 de enero de 1863 rumbo a la boca oriental del estrecho de Magallanes, llegando a bahía Posesión (52²17'S; 685' W) el 6 de febrero, partieron a Punta Arenas (5310'S; $70^{\circ} 54^{\prime} \mathrm{W}$ ) el 13 de 3 febrero, a la que llegaron el 14 de febrero, donde solo permanecieron un día "en esta ingrata región...donde está reunido todo lo malo que en la naturaleza se pueda concebir" (fide Martinic, 1991)1'; también, bahía Borja (53³3'S; $72^{\circ} 29^{\prime} \mathrm{W}$ ), puerto del Hambre (52 ${ }^{\circ} 39^{\prime} \mathrm{S}$; $70^{\circ} 55^{\prime} \mathrm{W}$ ) y bahía Gregorio (59³9'S; $\left.70^{\circ} 13^{\prime} \mathrm{W}\right)$, saliendo del

1 Memorándum de la navegación verificada en la Fragata de S.M.C. "Resolución", por el Mayor General de la Escuadra del Pacífico (...), en Ms. 808, Museo Naval, Madrid, el mes de Febrero de 1863.
Estrecho el 25 de febrero (Martinic, 1991).

A pesar de la insuficiente colaboración o nula colaboración de los marinos de la escuadrilla, el objetivo naturalista de la Comisión traducido en la recolección de "una cantidad regular de pieles de aves y mamíferos, insectos, conchas de moluscos, especímenes de plantas y otros elementos naturales". Estaciones de colecta fueron cabo Vírgenes (52¹9'S; $68^{\circ} 23^{\prime} \mathrm{W}$, República Argentina), Punta Arenas y alrededores, bahía San Juan de la Posesión (puerto del Hambre), bahía Borja y Playa Parda (podría ser 5319'S; 7301'W). Además, el gobernador de Punta Arenas cooperó con ellos obsequiándoles diversos especímenes y proporcionándoles cabalgaduras y guías (Martinic, 1991).

Los integrantes naturalistas con los que se inició la Comisión eran un Presidente (Patricio M. Paz y Membiela), un Jefe Científico (Fernando Amor, que falleció en el curso de la expedición en San Francisco, California), un naturalista (con funciones también de cronista y secretario, Francisco de Paula Martínez), un etnógrafo y antropólogo (Manuel Almagro y Vega), un botánico (Juan Isern), un dibujantefotógrafo (Rafael Castro) y un taxidermista (Bartolomé Puig) (Cabodevilla, 1998); fueron siete naturalistas y un fotógrafo, de los cuales solo cinco naturalistas capturaron insectos: $F$. Amor, P. Paz, Francisco de Paula Martínez y Sáez, Marcos Jiménez de la Espada y Juan Isern Batlló (Santos Mazorra, 1994).

El objetivo de esta Nota es analizar el aporte de la Comisión en lo referente a la recolección de insectos de Magallanes en esta región, ya que "los animales así, como las plantas del Estrecho de Magallanes, eran desde mucho tiempo bastante conocidos, i muchos de ellos mejor que los de Chile" y un gran número de expediciones navales científicas visitaron el Estrecho en tiempos en que la política de España no permitía que sus colonias fuesen visitadas por extranjeros" (Philippi, 1880).

Los antecedentes sobre los resultados de la Expedición proporcionados por Martinic (1991) en general y por Santos Mazorra (1994) en particular, fueron confrontados para establecer qué insectos se habían recolectado en las localidades del estrecho 
Tabla 1. Insectos de la región de Magallanes recolectados por la Comisión del Pacífico, todos con localidad "Magallanes (Chile)", y fecha cuando está registrada, cuyos componentes fueron extraídos del Catálogo de Santos Mazorra (1994)

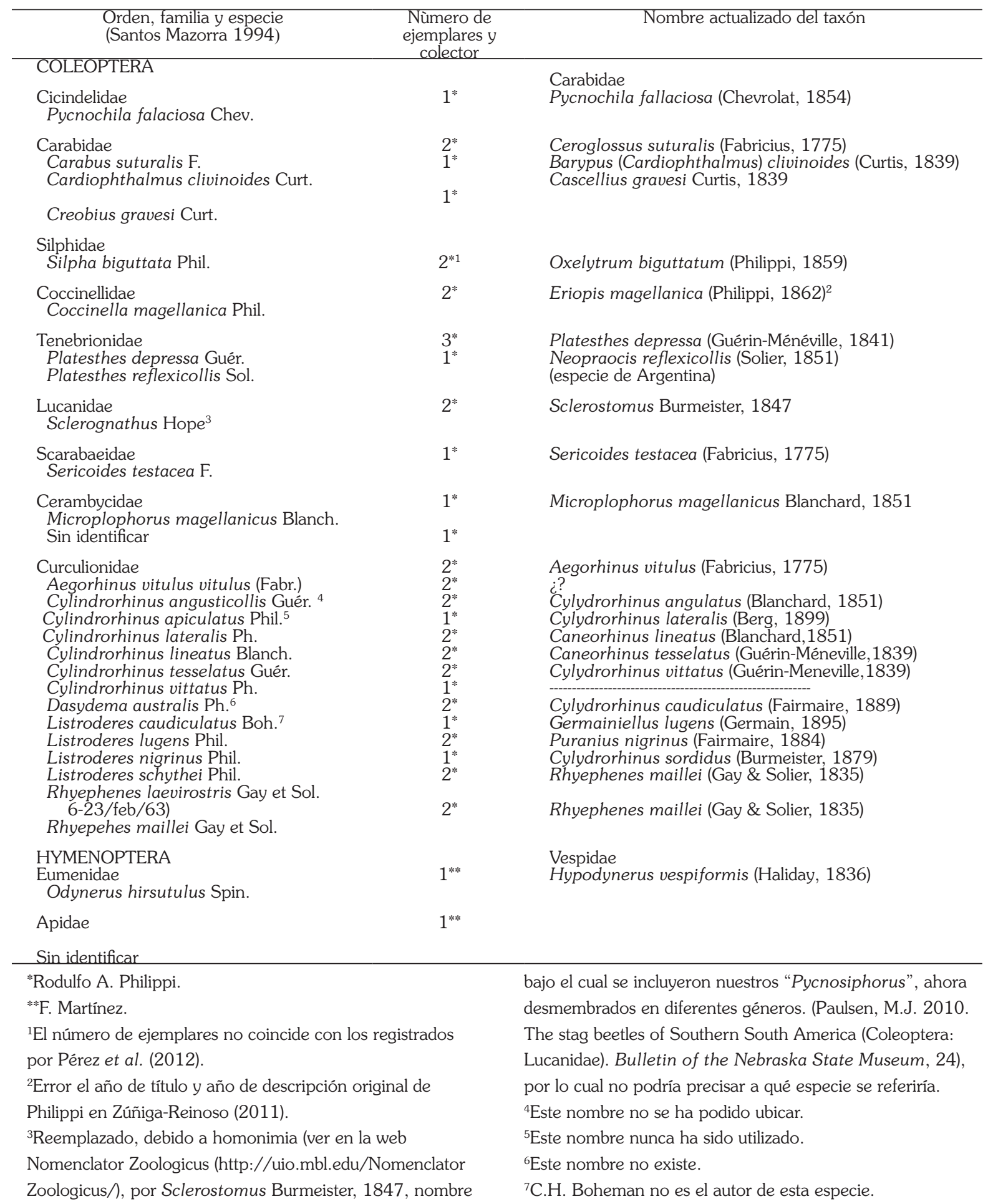


de Magallanes; además, dada la poca precisión de Santos Mazorra sobre las especies de insectos hizo necesario consultar las grandes compilaciones (Blackwelder, 1944a, 1944b, 1945, 1946, 1947, 1957), descripciones originales (Philippi 1862) y revisiones (Zúñiga-Reinoso, 2011; Pérez et al., 2012).

Santos Mazorra (1994) consigna que, entre los científicos y naturalistas que los expedicionarios conocieron en la región central de Chile y que les obsequiaron ejemplares de historia natural para añadirlos a las colecciones de la Comisión, se encontraba el "Dr. Roel Armando Philippi" (sic), en ese entonces Director del "Museo de Ciencias Naturales de Santiago de Chile", incluidos insectos, procedentes unos de su colección particular y otros de la institución que dirigía. El mismo autor, en su valoración de los resultados entomológicos de la Expedición anota que se muestreó la región del Sur de Chile, en la que se comprenden "Valdivia, Talcahuano y Colchagua", no mencionando en ningún momento Magallanes. $Y$ sin embargo, señala, en otro párrafo a F. Martínez como colector de Hypodynerus hirsutulus Spin. (= Hypodynerus vespiformis (Haliday, 1836), cuya localidad es Magallanes (Chile).

En ninguna parte de su Catálogo, el autor registra algún aporte de J. Schythe, el Gobernador de Magallanes de acuerdo a Martinic (1991).

En síntesis, la Expedición que se ha analizado, no significó ningún aporte al conocimiento de la entomología de la región de Magallanes, por las siguientes razones:

- No tuvo una definida orientación científica, ya que ésta solo estuvo subordinada a los intereses militares de la Escuadra, por motivaciones políticas. - A pesar del grupo científico claramente definido, los lugares visitados, de los cuales hemos agregado las coordenadas geográficas y las fechas de visita al Estrecho, no proporcionaron localidades definidas como para constituirse en antecedentes de distribución geográfica, por ejemplo.

- Prácticamente todos los ejemplares de Magallanes fueron proporcionados por don Rodulfo A. Philippi en Santiago. Solamente habría colectado en alguna localidad del Estrecho el español F. Amor.

- En el Catálogo de Santos Mazorra no hay ningún registro de ejemplares que habría obsequiado el Gobernador de Magallanes, Jorge Schyte (Martinic, 1991).

Los resultados científicos de la Expedición, destacados por Martinic (1991), Santos Mazorra (1994) y Puig-Samper (2003), no son del paso por el estrecho de Magallanes (1863) respecto a la entomología. Este hecho la diferencia de todas las expediciones extranjeras, de diferentes nacionalidades, que visitaron el territorio austral de Chile y que fueron la base de numerosas publicaciones científicas que incrementaron el conocimiento de la biodiversidad del extremo meridional de Sudamérica.

Hubo de transcurrir casi 130 años entre la publicación inicial de Almagro (1866) sobre las colecciones hechas y la de Santos Mazorra (1994) sobre los insectos recolectados.

Finalmente expresamos nuestros agradecimientos al colega Mario Elgueta, del Museo Nacional de Historia Natural de Santiago, Chile, por responder y aclarar las dudas referentes a la nomenclatura taxonómica de las especies.

Con todo, esta actualización que se presenta en la Tabla 1 tiene solo un valor relativo, ya que solo se abordó sobre el supuesto de que los nombres registrados por Santos Mazorra (1994) son válidos, ya que no aparece ningún especialista fundamentándolos.

\section{LITERATURA CITADA}

Alvarado Ballester, R. (1987). Ignacio Bolivar y Urrutia. Colección Ensayos. Fundación Juan March (Madrid). http://digital.march.es/ensayos/ fedora/repository/ensayos: 173/CBJ Blackwelder, R.E. (1944a). Checklist of the Coleopterous Insects of Mexico, Central America, the West Indies, and South America. Part 1. United States National Museum Bulletin, 185, i-xii, pp. 1-188. Blackwelder, R.E. (1944b). Checklist of the Coleopterous Insects of Mexico, Central America, the West Indies, and South America. Part 2. United States National Museum Bulletin, 185, [i-iv], pp.189-341. Blackwelder, R.E. (1945). Checklist of the Coleopterous Insects of Mexico, Central America, the West Indies, and South 
America. Part 3. United States National Museum Bulletin, 185, i-iv, pp. 343-55.

Blackwelder, R.E. (1946). Checklist of the Coleopterous Insects of Mexico, Central America, the West Indies, and South America. Part 4. United States national Museum Bulletin, 185, i-iii, pp. 551-763. Blackwelder, R.E. (1947). Checklist of the Coleopterous Insects of Mexico, Central America, the West Indies, and South America. Part 5. United States National Museum Bulletin, 185, i-iv, pp. 765-925.

Blackwelder, R.E. (1957). Checklist of the Coleopterous Insects of Mexico, Central America, the West Indies, and South America. Part 6. United States National Museum Bulletin, 185, i-vii, pp. 9271492.

Cabodevilla, M.A. (1998). Prólogo. Ecuador (1864-65) y la Comisión Científica del Pacífico. In Jiménez de la Espada, M., de Paula Martínez, F., M. Almagro \& J. Isern, El Gran Viaje. Editorial Abya-Yala. Quito. Ecuador.

Gomis Blanco, A. (Ed.) (1921). Ignacio Bolívar y las Ciencias naturales en España (Madrid). Imprenta Clásica Española. 211 pp. http://books.google.cl// books?id=45wBMu19_dUC\&printsec $=$ fro ntcover\&hl=es\# $\mathrm{v}=$ onepage\&q\&f$=$ false

Martínez-Valverde, C. (1958). Hernández Pinzón y Álvarez, L. In Martínez-Hidalgo y Terán, J.Ma., Enciclopedia General del Mar (Dir.). Tercer volumen. E-I., p.744. Ediciones Garriga S.A. MadridBarcelona.
Martinic. M. (1991). La "Comisión Científica del Pacífico" en Magallanes (1863). Anales del Instituto de la Patagonia, Serie Ciencias Naturales, 20:7-18.

Pérez, J., F. Prieto \& M. París (2012). Sílfidos iberoamericanos (Coleoptera, Silphidae) de la colección del Museo Nacional de Ciencias Naturales (CSIC) de Madrid (España). Arquivos Entomológicos, 7:5762.

Philippi, R.A. (1862). Sobre algunos insectos de Magallanes. Anales de la Universidad de Chile, 21:407-414. [RAE \& gestores]. 2012. Tecnicismos, neologismos $y$ extranjerismos en el español. http://www. $\mathrm{rae.es/rae/gestores/gespub000040.}$ nsf/(voanexos)/arch5AB7AFD22D 6B726DC1257B35004C7\$FILE/ TecnicismosNeologismosExtranjerismos. pdf

Puig-Samper, M. (1988). Crónica de una expedición romántica al Nuevo Mundo. CSIC. Madrid.

Santos Mazorra, C.M. (1994). Catálogo de los insectos recolectados por la Comisión Científica del Pacífico (1862-1865). Manuales Técnicos de Museología. Vol. 5. Museo Nacional de Ciencias Naturales. Consejo Superior de Investigaciones Científicas. Madrid. 196 pp.

Zúñiga-Reinoso, Á. (2011). Los Coccinélidos (Coleoptera: Coccinellidae) de la región de Magallanes. Nuevos registros y distribución regional. Anales del Instituto de la Patagonia, 39(1):59-71. 
